\title{
Helium settling and mass loss in magnetic Ap stars
}

\section{The chemical stratification}

\author{
S. Théado ${ }^{1,2}$, S. Vauclair ${ }^{3}$, and M. S. Cunha ${ }^{2}$ \\ 1 Institut d'Astrophysique et de Géophysique, Université de Liège, Allée du 6 Août 17, 4000 Liège, Belgique \\ e-mail: Sylvie.Theado@ulg.ac.be \\ 2 Centro de Astrofísica da Universidade do Porto, Rua das Estrelas, 4150-762 Porto, Portugal \\ ${ }^{3}$ Laboratoire d'Astrophysique, 14 av. Ed. Belin, 31400 Toulouse, France
}

Received 25 February 2005 / Accepted 4 August 2005

\section{ABSTRACT}

The chemical stratification is likely to play a predominant role in the excitation mechanism of high order modes in rapidly oscillating Ap stars. In this paper we discuss some processes (convection, He settling, mass loss and turbulent mixing) which may strongly affect the chemical composition of Ap stars. We discuss their interplay with a dipolar magnetic field in both magnetic polar and equatorial regions, where the magnetic lines are respectively mainly radially and horizontally oriented. We also present stellar evolutionary computations for magnetic Ap stars. As the magnetic geometry strongly breaks the sphericity of the star, we modeled and computed the polar and equatorial regions separately. We examine the effects of various chemical transport processes on the chemical stratification of both regions. In a forthcoming paper, we will study the stability of these models against pulsations.

Key words. diffusion - stars: abundances - stars: chemically peculiar - stars: interiors - stars: magnetic field - stars: mass loss

\section{Introduction}

The magnetic Ap stars are chemically peculiar stars which show overabundances of heavy elements and underabundances of some light elements. These abundance anomalies are distributed in patches and are believed to be confined in the external layers of the stars. Their distribution is related to the geometry of the magnetic field which is thought to be predominantly dipolar, although indications of deviations from dipolar structure are observed. The magnitudes of the magnetic fields range typically from a few hundred to a few thousand Gauss (e.g. Landstreet 1992; Mathys et al. 1997; Wade et al. 2000).

Among the coolest magnetic Ap stars, some have non-radial oscillations, (they are called "rapidly oscillating Ap stars" or roAp stars), while most of them, the noAp stars (for non-oscillating Ap stars) do not show any oscillation. Up to now, more than 30 roAp stars have been detected (Kurtz 1982; Martinez et al. 1991; Kurtz et al. 1994; Martinez et al. 1994; Girish et al. 2001; Elkin et al. 2005). Their oscillations are characterized by high frequencies, with periods between 4 and 16 min: they are interpreted as high order, low degree acoustic modes. These oscillations are different from the low frequency ones observed in the other types of oscillating stars which lie in the same region of the HR diagram. $\delta$-Scuti stars present low frequency acoustic modes which are due to $\kappa$-mechanism in the HeII ionisation region. This phenomenon is not present in
Am stars because of helium settling (Vauclair et al. 1974). On the other hand, $\gamma$-Doradus stars are the site of g-mode oscillations induced by "convective blocking" (Guzik et al. 2000). The specificity of roAp stars is related to the presence of a large magnetic field, which does not seem to be present in other stars.

Observations of the external parameters of roAp and noAp stars (the effective temperature and the luminosity) suggest that noAp stars are more evolved and more luminous than roAp stars (North et al. 1997; Handler \& Pauzen 1999; Hubrig et al. 2000). However, as discussed by Cunha (2002), an observational bias related to the frequency of the excited oscillations, which are expected to be lower in more evolved and more luminous stars, might prevent the detection of existing oscillations in the latter. Recently Elkin et al. (2005) have confirmed the existence of these luminous, evolved, lower frequency roAp stars, with the discovery of a 21 min pulsation in the cool magnetic Ap star HD 116114. Besides being evolved and luminous, compared to other roAp stars, HD 116114 does not show an anomalous relative abundance of the first and second ionization states of Pr and Nd. Such an anomaly has been found in all other roAp stars for which detail spectroscopic studies have been carried out, and is considered as the main spectroscopic discriminant between roAp and noAp stars (Gelbmann et al. 2000; Ryabchikova et al. 1904). Thus, it seems that the true differences between roAp and noAp stars are still far from being understood. 
The observational facts mentioned above point to an intricate relation between the surface chemical abundances, the presence or absence of magnetic fields and the properties of the oscillations excited, if any, in otherwise similar stars. The overall goal of this work is to improve our understanding of this intricate relation. In the present paper we will investigate possible equilibrium models of roAp stars, including different physical processes that might influence the chemical profiles in their envelopes. In principle, the different models can be indirectly tested, by a comparison of the properties of the pulsations that are predicted to be excited in each of them and the properties of the oscillations observed in roAp stars. This second step will be presented in a forthcoming paper.

In Sect. 2, we will discuss different physical processes that are expected to take part in the definition of the chemical profiles in the envelopes of roAp stars. Section 3 will be devoted to the physical inputs introduced in our stellar evolution computations: we will present the chemical transport processes included in our models, the adopted modeling and their interplay with the magnetic field. In Sect. 4 we will present the computational results; we will discuss the role of He settling and mass loss in the chemical composition of polar and equatorial regions of magnetic Ap stars in Sect. 5.

\section{Chemical stratification}

Main sequence cool A-type stars lie in the classical instability strip. Hence, at least a fraction of these stars is expected to pulsate, as result of the growing of perturbations, driven by the opacity mechanism. The excitation of oscillations in these stars is expected to depend on the abundance of some chemical elements, particularly in the regions where most of the driving and damping of the oscillations takes place. Chemical profiles not only determine whether oscillations are excited or not, but also they can introduce selective effects, influencing the properties of the modes that will be excited in a given star.

To understand why pulsations are found in some cool Ap stars but seem to be absent in others, or to understand why roAp stars pulsate in high frequencies only, while in $\delta$-Scuti stars only low frequency pulsations are excited, attention needs to be paid to the differences found when these classes of stars are compared. The chemical profiles are most likely one of such differences, and in particular one which can influence the pulsation properties of these stars.

Since the discovery of the first roAp stars (Kurtz 1982), several attempts have been made to explain the excitation of the observed oscillations. The observations were found rather puzzling, since stability analysis of standard models of stars in the region of the HR diagram where roAp stars are found predicted that only low frequency oscillations, like those observed in $\delta$-Scuti stars, would be unstable.

Different excitation mechanisms were proposed (see Balmforth et al. 2000 - hereafter BCDGV - for a review of these processes) and tested in a more or less extensive way. In particular, the importance that the chemical profiles might have in this context was first emphasized by Dolez \& Gough (1982), who pointed out that if helium were overabundant at the magnetic poles, it could eventually trigger the helium $\kappa$-mechanism at the poles and consequently drive non radial pulsations such as aligned axisymmetric modes. However the abundance anomalies observed in roAp stars are presumably due to element segregation which leads to helium depletion at the magnetic poles. This, however, could be compensated if the star were to lose mass predominantly at the magnetic poles (Dolez et al. 1988; Vauclair \& Dolez 1990). Vauclair et al. (1991) showed that in the presence of a small mass loss (about that of the solar wind) helium accumulates at the magnetic poles. In stars with effective temperatures lower than $18000 \mathrm{~K}$, this accumulation would not be visible as it would lie below the photosphere, but the suggestion was that it could still be sufficient to explain the pulsationnal instability.

Today, the most commonly accepted excitation mechanism in roAp stars is the $\kappa$-mechanism, acting in the region where the hydrogen is ionized (Dziembowski \& Goode 1996), rather than in the helium ionization region, where pulsations in $\delta$-Scuti stars are driven. However, only in non-standard stellar models with either an ad-hoc temperature inversion in the atmosphere (Gautchy \& Saio 1998), or the envelope convection suppressed (BCDGV, Cunha 2002) is that the driving by the $\kappa$-mechanism is found to be sufficiently efficient to compensate the energy that the high frequency modes lose in other regions of the star.

With this in mind, through the rest of this section we discuss convection, element segregation, mass loss and turbulence four physical processes that are determinant to the chemical stratification in the envelopes of Ap stars.

\subsection{Convection versus magnetic field}

Turbulent and convective motions may be strongly reduced in the presence of magnetic fields. The interaction between convection and magnetic field has been observed and studied for quite some time, particularly in the context of sunspots. In fact, the suppression of convection by the magnetic field in sunspots is generally accepted as the explanation for the lower temperatures observed in these regions of the sun's surface (e.g. Thomas \& Weiss 2004, for a review). A sufficient condition for suppression of convection in the presence of a magnetic field was derived analytically by Gough \& Tayler (1966), in a plane parallel approximation for a magnetic field not entirely horizontal, and extended to the case of a spherical shell by Moss \& Tayler (1969). These works (as well as later works by Shibahashi 1983 and by Cox 1984) indicate that if the field has a significant vertical component in the convectively unstable region, the onset of convection will be prevented even if the ratio of the magnetic to the gas pressure is relatively small there. However, no satisfying sufficient condition for stability could be derived for the cases in which the magnetic field has a horizontal section in the region of potential instability.

With these results in mind different authors have discussed models of roAp stars composed of a polar magnetic region, where convection was assumed to be either suppressed or to have a reduced efficiency, and a magnetic equatorial region where convection was considered not to be affected by the magnetic field (e.g. Dolez \& Gough 1982; Vauclair et al. 1991; BCDGV). 
However, as pointed out by Moss \& Tayler (1969), the problem of suppression of convection is not truly a local one. In fact, the linear theory can only guarantee that convection is suppressed in a spherical shell if such suppression takes place everywhere in that shell. Thus, the picture of convection being suppressed in the regions where the magnetic field is closer to vertical, whilest being maintained in the regions where it is horizontal, might not hold once convection is fully developed in the latter.

The fact that no condition for stability could be derived for regions where the magnetic field has a horizontal section in the spherical shell does not necessarily mean that convection cannot be suppressed (or its efficiency largely reduced) there. Generally, such suppression of convection will depend on how localized the perturbation is. If the layer which is convectively unstable is very thin and the perturbations well localized (e.g. non-zero only in the convectively unstable region), then it is possible to estimate the horizontal magnetic field needed to suppress convection (Moss \& Tayler 1969) through the condition:

$\frac{B^{2}}{\gamma P}>\frac{4}{\gamma \pi^{2}}\left(\frac{d}{H_{\mathrm{p}}}\right)^{2} \delta\left(\nabla-\nabla_{\mathrm{ad}}\right)$

where $B$ is the magnitude of the magnetic field, $P$ is the gas pressure, $\gamma$ is the first adiabatic exponent, $H_{\mathrm{p}}$ is the pressure scale hight, $\delta=-[\partial \ln \rho / \partial \ln T]_{\mathrm{P}}, \nabla=\partial \ln T / \partial \ln P, \nabla_{\mathrm{ad}}=$ $[\partial \ln T / \partial \ln P]_{\mathrm{ad}}$, "ad" means adiabatic changes and $\rho$ and $T$ are the density and temperature, respectively; $d$ is related to the length, along the magnetic field line, over which the perturbation is non-zero. For perturbations confined in the convectively unstable region $d$ can be estimated by the relation $d=\left(r_{0} \tilde{d}\right)^{1 / 2}$, where $\tilde{d}$ is the thickness of the convectively unstable region and $r_{0}$ is the radius at which the latter is located.

Rapidly oscillating Ap stars offer optimal conditions for suppression of convection. Typically the convectively unstable layer in the envelope is rather thin and $B^{2} / P$ is relatively large there. In Fig. 1 we show the pressure scale height, $H_{\mathrm{p}}$, versus $\log T$, where $T$ is the temperature, for a model with 2 solar masses and 472 Myrs of age, together with the thickness of the convectively unstable layer. The convectively unstable layer in the envelope of this model is associated with the region of hydrogen ionization and is indeed rather thin, with $\tilde{d} \approx H_{\mathrm{p}}$ in that region. In Fig. 2 we show the right hand-side of Eq. (1) for the same model and the corresponding left hand-side, assuming a magnetic field $B=3000$ Gauss. In this case the condition expressed by Eq. (1) is verified and we might expect that so far as the perturbations are confined to the unstable layer, convection might be suppressed even if the magnetic field is horizontal.

In real stars there are no walls confining the perturbation to the convectively unstable layer, and thus perturbations on larger scales might develop which cannot be suppressed by the horizontal magnetic field. However, such perturbations on larger scales would permeate the adjacent angular regions, where the magnetic field has a vertical component, and thus could perhaps be suppressed by the action of the magnetic field there.

The above discussion reiterates the difficulties faced when having to decide on theoretical grounds whether and where

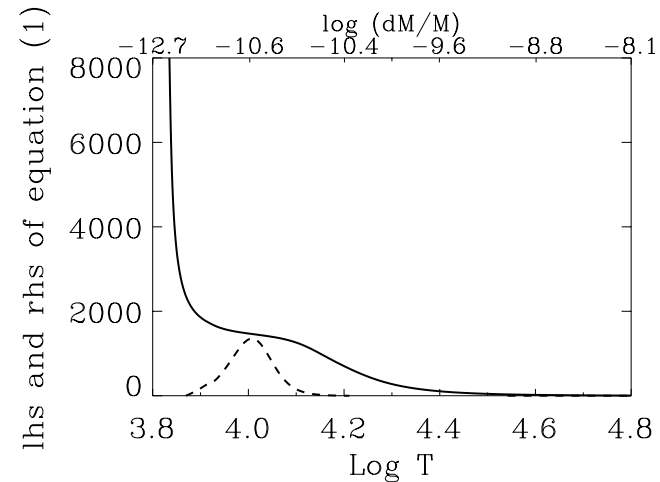

Fig. 1. For a model of mass $M=2 M_{\odot}$ and age $=472 \mathrm{Myrs}$, the figure shows: dotted line - pressure scale hight, $H_{\mathrm{p}}$, versus the logarithm of temperature; continuous line - same as dotted line but in the convectively unstable region only; dashed line - thickness of the convectively unstable region in the envelope

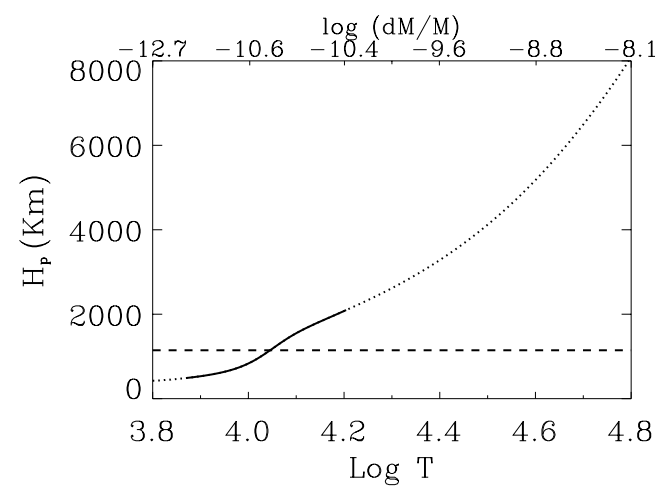

Fig. 2. For the same model as in Fig. 1 the figure shows: continuous line - left hand-side (lhs) of Eq. (1) assuming a magnetic field with a magnitude of 3000 Gauss; dashed line - the right hand-side (rhs) of the same equation.

convection is suppressed by the magnetic field in the envelope of Ap stars. Indirect support for suppression of convection at least in some angular region of cool Ap stars comes also from stability analysis of pulsations in models of roAp stars. In models with envelope convection suppressed high frequency oscillations are found to be intrinsically unstable (BCDGV, Cunha 2002) and the theoretical predictions are generally in good agreement with observations. Moreover, if convection were present in the equatorial region while suppressed at the poles, modes aligned with the magnetic field would be preferentially excited. Such alignment, however, is only expected if the magnetic field of the star is sufficiently large that its effect on the oscillations is much greater than the effect of the centrifugal distortion of the star (Bigot \& Dziembowski 2002).

In principle additional information about the suppression of convection might also be inferred from the observations. In cool Ap stars convection is expected to take place in and below the atmosphere. Thus, the turbulence associated with convection should contribute to the broadening of the spectral lines. Velocity fields associated with convection have been detected in non-magnetic stars with very sharp lines, in the temperature range in which roAp stars are found (Landstreet 1998). Moreover these velocity fields introduce an asymmetry in the 
lines which is clearly detected in these stars. Some cool Ap stars, with low magnitude magnetic fields, have spectral lines sufficiently sharp that such an asymmetry introduced by convection should be seen. However, a first look at the data seems to indicate that in most cases the lines of these cool Ap stars do not show that kind of asymmetry (Landstreet - private communication). This favors the idea that even a relatively small magnetic field in the atmosphere might suppress convection at all latitudes.

From the above discussion we feel that the extent to which convection is suppressed by the magnetic field in the envelopes of Ap stars is not easily deduced from present theoretical and/or observational information. Therefore, in the present work convection in the envelope of Ap stars is assumed to be suppressed at all latitudes. We hope that future stability analysis of the models presented here, in combination with observations of pulsations in roAp stars, will allow for indirect tests on this assumption.

\subsection{Element segregation}

Microscopic diffusion including gravitational settling and radiative levitation is known to be a basic process acting in the radiative interiors of most main-sequence stars. Its effects on magnetic A stars have been discussed many times in the past (Chapman \& Cowling 1970; Michaud et al. 1981; Dolez \& Gough 1982; Dolez et al. 1988) and the expected effects of diffusion may be summarized in the following way. The main effect of microscopic diffusion on the stellar structure is that helium falls down towards the central regions of the star while hydrogen goes up for hydrostatic balance. Meanwhile most heavy elements are pushed upwards by the radiative force and may accumulate in the very high atmosphere, giving rise to abundance clouds as detected, for example, by Ryabchikova et al. (1904). According to Richard et al. (2001), the radiative acceleration also leads to the accumulation of iron-peak elements around $200000 \mathrm{~K}$. This accumulation results in a substantial opacity increase which may lead to the appearance of a convectively unstable region. As described by Turcotte et al. (2000), this phenomenon may affect the stability of the star and especially the excitation of low frequency modes. As described in BCDGV, the excitation of higher frequency modes such as those observed in roAp stars mostly depends on the helium and hydrogen abundances just below the photosphere, where the abundances of metals are not expected to vary in a significant way. In the following, we neglect metal diffusion.

In a magnetic field, the diffusion-induced motions of ions across the magnetic lines are slowed down. This has been described in Vauclair et al. (1979) and Michaud et al. (1981). Between two collisions, ions spiral along the magnetic lines: this leaves the component of the diffusion velocity parallel to the magnetic lines unchanged but alters the velocity component perpendicular to the magnetic field. This component is decreased by a factor $\left(1+\omega^{2} t^{2}\right)$ where $t$ is equal to the collision time for test atoms and $\omega$ is the period of the spiral rotation of the ions around the magnetic lines:

$t=\frac{m_{i} D_{i}}{k T}$ where $m_{i}$ is the mass of the diffusing particle, $D_{i}$ the diffusion coefficient, $k$ the Boltzmann constant and $T$ the temperature; $\omega$ may be written:

$\omega=\frac{Z e B}{m_{i} c}$

where $Z e$ is the charge of the particle (in e.s.u), $B$ the strength of the magnetic field and $c$ the light velocity in vacuo.

Let us examine the magnetic field effects on helium settling in the external layers of the star where most of the driving and damping is thought to take place (cf. Sect. 2). Just below the stellar surface, helium is mostly neutral, therefore it does not undergo the effect of magnetic field. In the region of He first ionization, we obtain from models: $Z \simeq 1, D_{\mathrm{He}} \simeq 10^{4} \mathrm{~cm} \mathrm{~s}^{-1}$ and $T \simeq 10^{4} \mathrm{~K}$. For a $2000 \mathrm{G}$ horizontal magnetic field (which is a typical value near the magnetic equator of roAp stars) this leads to a very small value of $(\omega t)^{2}$ (around 0.05 ). In these conditions we expect He settling to be hardly reduced by the horizontal magnetic field in the regions where the oscillations are excited. This result is consistent with Fig. 1 of Vauclair et al. (1991) which displays the horizontal component of the magnetic field required to prevent diffusion: in the external layers of the star only a magnetic field larger than $10^{5}$ would prevent helium settling.

\subsection{Mass loss}

Outflows of mass in the form of stellar winds have been detected by a variety of means throughout the Hersprung-Russell diagram both on and above the main sequence (Cassinelli \& MacGregor 1986; Cassinelli \& Lamers 1987; Dupree \& Reimers 1987). Stellar winds seem to be nearly ubiquitous in every spectral type stars and every evolutionary stages.

The winds from hot and cool main-sequence stars seem to fall into distinct categories. The winds emanating from hot luminous stars are characterized by high rates of mass loss $\left(\dot{M} \simeq 10^{-6} M_{\odot} \mathrm{yr}^{-1}\right)$ and high asymptotic flow speeds ( $\simeq 3 u_{\text {esc }}$ where $u_{\text {esc }}$ is the surface gravitational escape speed) (Charbonneau \& MacGregor 1995). By analogy with the Sun, winds from solar type stars are thought to have a moderate asymptotic speed $\left(\simeq u_{\mathrm{esc}}\right)$ and low mass rates $(\dot{M} \simeq$ $\left.10^{-14} M_{\odot} \mathrm{yr}^{-1}\right)$. An abundance of observational evidence suggests that winds from hot luminous stars are radiatively driven, while winds from solar type stars are presumably primarily thermally driven and linked to the presence of a high temperature corona. However Alfvén waves and shock waves (generated by the stellar convection or radial pulsations) have also been proposed as contributors to the driving of the winds (e.g. Holzer 1987; Holzer \& Macgregor 1985). Alfvén waves have been invoked as a means of further accelerating the stellar winds to larger asymptotic speeds, with applications to high speed solar wind streams (Jatenco-Pereira et al. 1994) and hot stars winds (Underhill 1983; dos Santos et al. 1993), while shock waves may be important in heating the chromosphere (Holzer 1987; Cuntz 1990).

The magnetic Ap stars belong to a spectral type intermediate between hot and solar type stars. Unfortunately the mechanism and the properties of their mass loss are poorly known. 
Several measurements failed to detect any corona in these stars and suggest a thermal origin for their emission (Drake et al. 1987; Willson et al. 1988). This seems to exclude the presence of a high temperature corona and thus of a thermally driven wind. On the other hand the expected mass loss rate driven by stellar radiation in such stars is small (Babel \& Montmerle 1997). Very few constraints exist on the mass loss rate of A stars and both radio measurements (Brown et al. 1992) and $\mathrm{H}_{\alpha}$ asymmetry investigation (Lanz \& Catala 1992) gives only an upper limit of $10^{-10} M_{\odot} \mathrm{yr}^{-1}$. The existence of the abundance anomalies implies a still smaller value lower than about $10^{-12} M_{\odot} \mathrm{yr}^{-1}$ (Michaud 1986). Michaud et al. (1983) showed that a mass loss of order $10^{-15}$ to $10^{-14} M_{\odot} \mathrm{yr}^{-1}$ may provide a quantitative agreement between computed and observed abundances in Am stars. This result was confirmed later by Babel \& Michaud (1991).

Hot magnetic chemically peculiar stars show evidence of anisotropic winds, as indicated by spectral observations of UV lines (Shore et al. 1987; Shore \& Brown 1990). This anisotropy is thought to result from interactions between the radiatively driven wind from the star and the magnetic field. Among these stars, He-strong and He-weak stars present sometimes signs of magnetically controlled winds (Brown et al. 1985; Shore \& Brown 1990) which have been interpreted as the signature of mass loss near one magnetic pole. For Herich stars, a stellar wind has also been invoked by Vauclair (1975) to account for their observed helium enrichment (by a factor 2 or 3 ): this enrichment cannot be explained by diffusion alone as the upward radiative acceleration on helium is always smaller that the gravity (Montmerle \& Michaud 1976; Michaud et al. 1979). Vauclair (1975) showed that an overall macroscopic mass loss flux could advect helium up to the observed layers. These stars are of particular interest for magnetic Ap stars studies because even if the mechanism and the origin of the wind is not known in Ap stars, we expect some analogies with the wind of He-weak and He-rich stars due to their similar magnetic characteristics. If a wind is present in Ap stars, it must have a geometry mainly determined by the magnetic field geometry and larger mass loss rates must be present in the polar region than at the magnetic equator. These results have been confirmed by theoretical studies which show that a stellar wind in the presence of a dipolar magnetic field can freely flow only from the polar regions, where the magnetic field lines are open (Shore 1987; Babel \& Montmerle 1997). The wind then forms two polar jets, whereas at the latitudes near the magnetic equator the wind is inhibited and the matter is trapped, forming the so-called "dead zone".

\subsection{Turbulent transport}

Turbulent transport is likely to occur in the radiative interiors of non-magnetic stars (see Zahn 1993, and references therein). These motions may arise from the occurrence of various hydrodynamical instabilities related or not to the rotation.

\subsubsection{Rotation-induced mixing}

In a rotating stars, due to centrifugal effects, the gravity equipotentials are no longer spherical. This induces a circulation of matter between the polar and the equatorial regions: the socalled meridional circulation. This circulation itself induces a transport of angular momentum, thereby creating shears which become unstable in the horizontal direction while they are stabilized by the density stratification in the vertical direction. The coupling between circulation and turbulence leads to a mixing which may be efficient in inhibiting the other involved chemical transport processes.

\subsubsection{Thermohaline instabilities}

Among the hydrodynamical instabilities which are not related to the presence of rotation, one is likely to occur in the case of stars with stellar winds: the thermohaline instability. In the presence of stellar wind, helium should accumulate in a narrow region where the total velocity (including the diffusion velocity and the wind-induced velocity) vanishes. As a consequence a helium rich layer may appear above hydrogen rich regions. This situation is unstable: thermohaline instability develops in the star and leads to a special kind of convection whose efficiency is difficult to evaluate (see Vauclair 2004, and references therein).

\subsubsection{Turbulence versus magnetic field}

Turbulent motions as well as convective motions may be strongly reduced in the presence of magnetic field, at least in the polar regions of the stars (cf. Sect. 2.1). However, according to Vauclair et al. (1991), the thermohaline mixing cannot be completely prevented by a magnetic field, because if it were so the helium accumulation observed in He-rich stars would be much more severe. This can be qualitatively understood as, contrary to dynamical convection, thermohaline convection cells have the shape of radial fingers which may still develop along the vertical magnetic lines. Following Vauclair et al. (1991), we will assume that in the polar regions the magnetic field reduces the turbulent motions to a mild mixing when helium accumulates above hydrogen rich regions. In the equatorial regions, as the wind is suppressed by the magnetic field no thermohaline instabilities will arise. Turbulent motions are then primary due to rotation-induced mixing. As for convection, the issue concerning the occurrence of turbulence in the equatorial regions is not settled. We will then treat two extreme cases: models will be computed assuming no turbulence in the equatorial regions and models will include a turbulent mixing in the vicinity of the equator.

\section{Physical inputs and structure of our models}

As described in Sect. 2, the dipolar magnetic structure of magnetic Ap stars strongly affects their envelope. While some physical processes which are otherwise supposed to occur in Ap stars are suppressed in polar or equatorial regions, others 
proceed freely or are only slightly altered. This leads to different chemical composition in polar and equatorial regions.

Following Vauclair et al. (1991), the star is schematically divided in two regions: the polar region where the magnetic field is primarily radially oriented and the equatorial region where it is primarily horizontal. These two regions are modeled and computed separately. The polar and equatorial models are then patched together below the base of the convective zone to form a composite model (as described by Balmforth et al. 2001) whose stability will be studied in a forthcoming paper (Cunha et al. 2005).

Our models are computed with the Toulouse-Geneva evolutionary code using the following physical parameters:

- opal equation of state (Rogers \& Nayfonov 2002);

- opal opacities (Iglesias \& Rogers 1996) completed by the

Alexander \& Ferguson (1994) low temperature opacities;

- NACRE nuclear reaction rates (Angulo et al. 1999).

The opacities are computed for each time step in each shell by taking into account the chemical composition variations. A more detailed description of the code can be found in Charbonnel et al. (1992), Richard et al. (1996) and Richard et al. (2004). In the following, we describe the adopted modeling for the main physical processes included in our models and we present the way the effects of magnetic field are introduced in the computations.

\subsection{Microscopic diffusion}

The chemical composition variations induced by diffusion are computed by solving at each time step, along a stellar evolution track, the following equations (cf. Charbonnel et al. 1992). For a species $i$ :

$\frac{\partial c_{i}}{\partial t}=D^{\prime}{ }_{1 i} \frac{\partial^{2} c_{i}}{\partial m_{r}^{2}}+\left(\frac{\partial D^{\prime}{ }_{1 i}}{\partial m_{r}}-V^{\prime}{ }_{1 i}\right) \frac{\partial c_{i}}{\partial m_{r}}-\left(\frac{\partial V_{1 i}^{\prime}}{\partial m_{r}}+\lambda_{i}\right) c_{i}$

with

$D^{\prime}{ }_{1 i}=\left(4 \pi \rho r^{2}\right)^{2} D_{1 i}$

and

$V^{\prime}{ }_{1 i}=\left(4 \pi \rho r^{2}\right) V_{1 i}$

where $m_{r}, r$ and $\rho$ are respectively the mass, the radius and the density at the considered point; $c_{i}$ is the concentration of species $i ; \lambda_{i}$ the nuclear reaction rate; $D_{1 i}$ is the molecular diffusion coefficient of element $i$ with respect to element 1 , which is traditionally hydrogen; $V_{1 i}$ is the diffusion velocity of element $i$ with respect to element 1 .

The diffusion velocity is computed in the test atom approximation:

$V_{1 i}=D_{1 i}\left\{-\nabla \ln c_{i}+k_{p} \nabla \ln p+\alpha_{1 i} \nabla \ln T-\frac{m_{i} f_{i}}{k T}\right\}$

where $p$ is the pressure, $T$ the temperature, $k$ the Boltzmann constant, $\alpha_{1 i}$ is the thermal diffusion factor, $k_{p}$ is a coefficient which will be expressed below, $f_{i}$ the radiative acceleration on the considered element and $m_{i}$ its mass. In the case of helium the radiative acceleration is always negligible because the helium cosmic abundance is very high. Moreover, in the considered stars, the thermal diffusion is also usually negligible (Michaud et al. 1979).

Taking into account the electrical field induced by ionelectron separation, the gravitational settling term can be written:

$k_{p} \nabla \ln p=\left(A_{i}-\frac{Z_{i}}{2}-\frac{1}{2}\right)\left(\frac{m_{\mathrm{H}}}{k T} \frac{G m_{r}}{r^{2}}\right)$

where $A_{i}$ and $Z_{i}$ stand respectively for the atomic mass and the charge of the considered element and $m_{\mathrm{H}}$ for the hydrogen atomic mass.

The diffusion velocity for partially ionized elements is written as:

$V_{1 i}=\sum_{i} V_{1 i}(k) x_{i}(k)$

where $V_{1 i}(k)$ is the diffusion velocity of a particle $i$, in a state of ionization $k$ and $x_{i}(k)$ the ionization fraction for this particle ionized $k$ times. The ionization fractions are computed using Saha equation where the partition functions are limited to the statistical weight of the ground levels.

In the case of fully ionised plasma, the interaction between two charged particles can be described by the static screened Coulomb potential of the Debye Huckel type. In this case, the diffusion coefficient $D_{1 i}$ is computed using the Paquette et al. (1986) formalism.

In the case of partially ionized medium, the Coulomb potential is not adequate to describe the interaction between particles. To describe interaction between a proton (hydrogen is supposed to be fully ionized) and a neutral atom, we use relation 8 of Michaud et al. (1978):

$D_{1 i}=\frac{3.68 \times 10^{21}}{n_{\mathrm{p}}}\left(\frac{T_{4}\left(1+A_{i}\right)}{A_{i}}\right)^{\frac{1}{2}} \frac{1}{\Omega(1,1)^{*}}$

with $\Omega(1,1)^{*}$ given in their Table 1 . Here $T_{4}$ is the temperature in units of $10^{4} \mathrm{~K}$ and $n_{\mathrm{p}}$ is the proton density number.

As discussed in Sect. 2.2, the excitation of high frequency modes mostly depends on the $\mathrm{He}$ and $\mathrm{H}$ abundances. Therefore we focus in this paper on the chemical stratification of these two elements and we only compute the diffusion of helium with respect to hygrogen. Metal diffusion as a whole will be studied in a forthcoming paper.

\subsection{Stellar wind}

For models including a mass loss, the outgoing flux is modeled by adding to the diffusion velocity an extra term:

$V^{\prime}{ }_{1 i}=\left(4 \pi \rho r^{2}\right)\left(V_{1 i}+\frac{\Gamma}{\rho}\right)=\left(4 \pi \rho r^{2}\right) V_{\text {tot }, i}$.

The $\Gamma$ term, which represents the outward helium flux due to the wind, is considered as a free parameter characterizing the strength of the wind. The mass loss rate can then be expressed as a function of $\Gamma$ :

$\dot{M}=4 \pi R^{2} \Gamma$

where $R$ is the radius of the star. 


\subsection{Turbulent transport}

In our computations, turbulent transports are modeled as pure diffusive processes by simply adding a turbulent diffusion coefficient in the diffusion Eq. (5), $D^{\prime}{ }_{1 i}$ becomes:

$D^{\prime}{ }_{1 i}=\left(4 \pi \rho r^{2}\right)^{2}\left(D_{1 i}+D_{\text {eff }}\right)$

where $D_{\text {eff }}$ is a global turbulent diffusion coefficient including the contribution of all involved turbulent transport processes: the rotation-induced mixing $\left(D_{\text {rot }}\right)$ and thermohaline instabilities $\left(D_{\text {turb }}\right)$.

\subsubsection{Rotation-induced mixing}

For models including rotational mixing, the turbulent diffusion coefficient $D_{\text {rot }}$ will be a free parameter. It will be chosen large enough to be able to homogenise the radiative interiors in the equatorial regions and thereby to prevent helium from falling down towards the central regions.

\subsubsection{Thermohaline instabilities}

Because of the stellar wind, a helium rich layer may appears above hydrogen rich regions. As described in Sect. 2.4.2, thermohaline instabilities may then arise. These instabilities are introduced in our computations in the following way: the stellar gas is assumed stable above the He peak thanks to the stable molecular weight stratification. Below the peak, we parametrize the mixing with an exponential turbulent diffusivity:

$D_{\text {turb }}=D_{\max } \exp \left(\ln 2 \frac{r-r_{\max }}{\Delta}\right)$

where $D_{\max }$ and $r_{\max }$ are respectively the value of $D_{\text {turb }}$ and the radius at the He peak; $D_{\max }$ is a free parameter. The parameter $\Delta$ represents the half width of the mixed region: it is chosen so that at the radius $r=\left(r_{\max }-\Delta\right)$ the molecular weight gradient is lower than a critical value arbitrarily chosen as:

$\nabla_{\mu}=\frac{\mathrm{d} \ln \mu}{\mathrm{d} r}<4 \times 10^{-11} \mathrm{~cm}^{-1}$.

\subsection{Magnetic field}

The effects of the magnetic field are introduced in the computations in an indirect way through its effects on the involved chemical transport processes and hydrodynamical instabilities.

Following the discussion in Sect. 2.1, convection is suppressed in both polar and equatorial models.

As described in Sect. 2.3, mass loss is supposed to be suppressed by the magnetic field in equatorial regions. As a consequence only polar models will be computed with mass loss.

Microscopic diffusion occurs freely in polar models. In equatorial models we take into account the decrease of the diffusion velocity due to the horizontal magnetic field although we expect this decrease to be very small for helium (as discussed in Sect. 2.2).

In polar models the magnetic field is supposed to be able to suppress the rotation-induced mixing but a mild mixing is

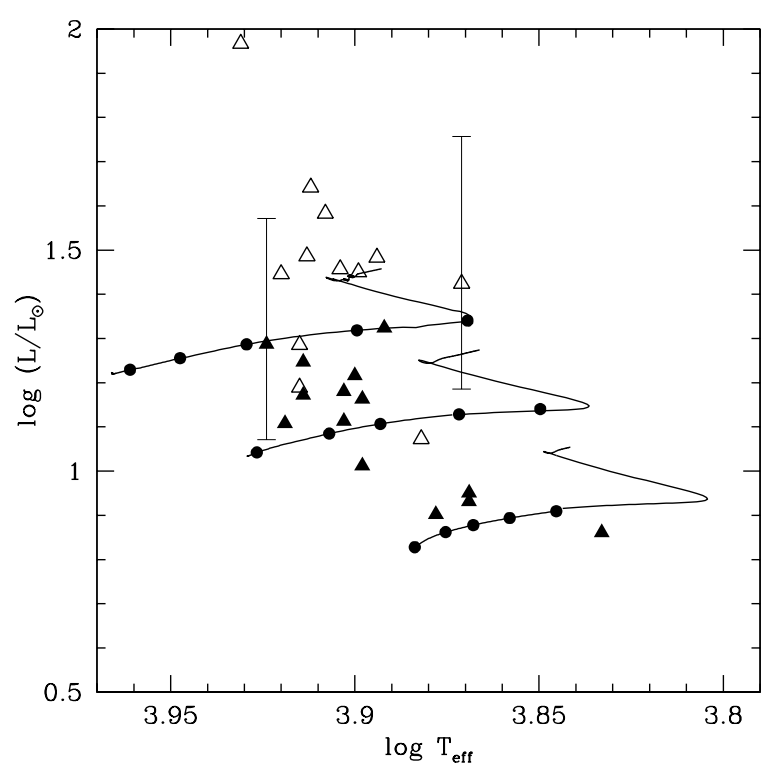

Fig. 3. HR diagram for polar models including He settling but no wind. For each evolutionary track, the solid circles show the position of models at different ages; for $1.6 M_{\odot}: 88$ Myrs, 479 Myrs, 675 Myrs, 885 Myrs, 1095 Myrs; for $1.8 M_{\odot}: 88$ Myrs, 479 Myrs, 675 Myrs, 885 Myrs, 1032 Myrs and for $2.0 M_{\odot}: 88$ Myrs, 274 Myrs, 479 Myrs, 675 Myrs, 824 Myrs. Observations by North et al. (1997) are also represented: full triangles display 14 roAp stars and open triangle display 12 noAp stars. For clarity we do not show all error bars but only the largest ones for roAp and noAp stars.

introduced to reproduce the effects of thermohaline convection (with small turbulent diffusion coefficient). For equatorial regions, two cases are treated: equatorial models which are computed with element segregation and no turbulence and equatorial models which include element segregation as well as a strong mixing.

\section{Computations}

We computed stellar evolutionary models for three masses: 1.6 $M_{\odot}, 1.8 M_{\odot}$ and $2.0 M_{\odot}$. For each mass both polar and equatorial models have been computed. These models include the physical processes and modeling discussed in Sect. 3. We recall that convection is suppressed in all our models.

\subsection{Polar models}

In this section we first present models including only element segregation. Then we will show models including element segregation, stellar wind and the induced thermohaline instabilities.

\subsubsection{Helium settling}

Figure 3 shows the evolutionary tracks of 3 models (from bottom to top $1.6 M_{\odot}, 1.8 M_{\odot}$ and $2.0 M_{\odot}$ ) including helium settling but no wind. The solid circles present the position on the HR diagram of models at different evolutionary steps. 

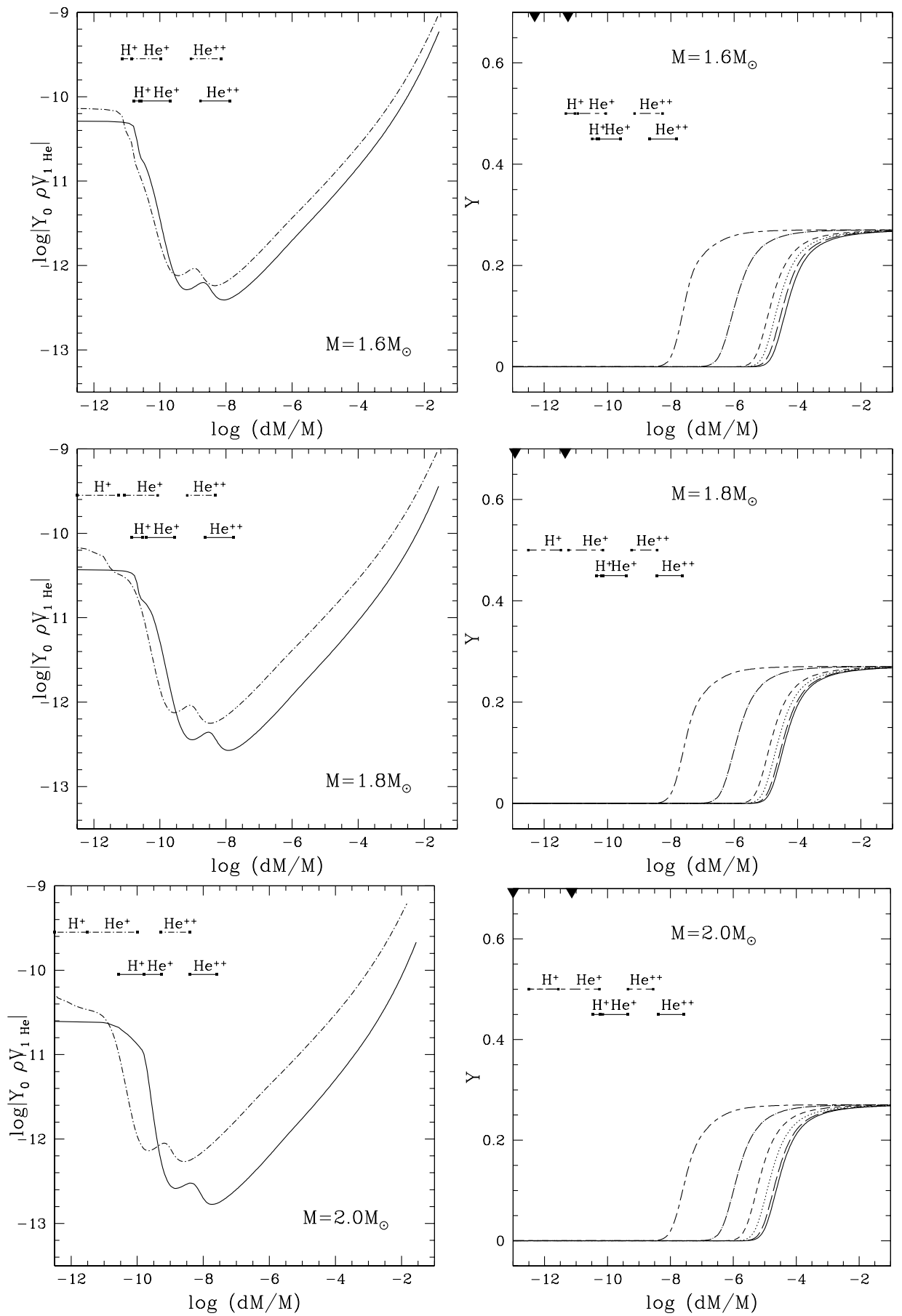

Fig. 4. Polar models including He settling but no wind. Left column: original helium diffusion flux ( $Y_{0}$ is the initial He mass fraction, $V_{1 \mathrm{He}}$ is the diffusion velocity of helium and $\rho$ the density, $\left|Y_{0} \rho V_{1 \mathrm{He}}\right|$ is given in cgs units) versus the outer mass fraction in the star for two ages

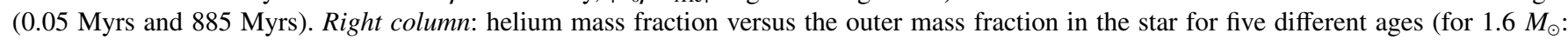
9 Myrs, 88 Myrs, 479 Myrs, 675 Myrs, 885 Myrs, 1095 Myrs; for $1.8 M_{\odot}$ : 9 Myrs, 88 Myrs, 479 Myrs, 675 Myrs, 885 Myrs, 1032 Myrs; for 2.0 $M_{\odot}: 9$ Myrs, 88 Myrs, 274 Myrs, 479 Myrs, 675 Myrs, 824 Myrs.). We have represented on each plot the hydrogen and the two helium ionization regions for the first and the last ages, the limits of the regions are given for fractions of neutral hydrogen or helium of $95 \%$ and $5 \%$ and for the same fractions of singly ionized helium. The solid triangles on the upper scale represent the depth where the optical depth is equal to 1 for respectively the youngest (on the left) and the oldest model (on the right).

In Fig. 4, the left column displays the original helium diffusion flux computed for homogeneous abundances (logarithm of diffusion velocity multiplied by density and by initial $\mathrm{He}$ mass fraction) versus the outer mass fraction for two evolutionary stages (0.05 Myrs and 885 Myrs) of the models. For both ages the general behaviour is the same but the position of the curves changes with the position of the helium ionization regions. In the left part of the curves, helium is mostly neutral: as the helium atoms suffer no Coulomb forces, the diffusion flux is high. The sharp decrease observed downwards (to the 
right) is mainly due to the decrease in the abundance of neutral helium. When helium is singly ionized, the diffusion flux increases as $T^{3 / 2}$. In the He second ionization region, the flux decreases again and increases finally like $T^{3 / 2}$ when He is completely ionized (see Vauclair 1975; Vauclair et al. 1991).

The right column of Fig. 4 presents the He profiles inside the three models at different epochs (after 9 Myrs on the ZAMS and at the five epochs represented by the solid circles in Fig. 3). As expected, microscopic diffusion rapidly drains helium off the helium and hydrogen ionization regions. Helium being replaced by hydrogen, these regions become rapidly hydrogen-rich.

Diffusion velocities are computed in the assumption of fully ionized hydrogen (cf. Sect. 3.1). According to Fig. 4 the region where hydrogen is mostly neutral is extremely thin and confined in the very external layers of the star. According to the He profiles, this regions become rapidly helium poor (the He abundances fall to zero before 1 Myrs in all our models). A more accurate treatment of diffusion coefficients would consist in taking into account the collisions between neutral hydrogen and helium atoms. This would lead to greater He diffusion flux in the external layers of the stars and would drain $\mathrm{He}$ off the external layers still more rapidly. As helium already diffuses out of these regions extremely rapidly, the approximation of fully ionized hydrogen probably does not alter the results in a significant way.

\subsubsection{Mass loss}

In this section we present computations including both helium settling and mass loss. The outward helium flux due to the wind is determined through the free parameter $\Gamma$, chosen so that the wind-induced helium accumulation does not lie at the surface but deeper in the stellar interior. This choice is motivated by the fact that helium is underabundant in all Ap stars where it can be observed (Ryabchikova 1991, and references therein).

The turbulent diffusion coefficient $D_{\text {turb }}$ due to the thermohaline mixing is chosen so that the local helium overabundances (in the helium peak) are consistent with those observed in helium rich stars (factor of 2 to 3 maximum).

For each mass, we compute two models: one model with $\Gamma=10^{-11} \mathrm{~g} \mathrm{~cm}^{-2} \mathrm{~s}^{-1}$ and $D_{\text {turb }}=2 \times 10^{5} \mathrm{~cm}^{2} \mathrm{~s}^{-1}$, one model with $\Gamma=3 \times 10^{-11} \mathrm{~g} \mathrm{~cm}^{-2} \mathrm{~s}^{-1}$ and $D_{\text {turb }}=10^{6} \mathrm{~cm}^{2} \mathrm{~s}^{-1}$.

The evolutionary tracks of these models are very similar to those of models without wind (Fig. 3).

As the stellar structure of our models changes during the evolution, their mass loss is not constant along the mainsequence. Table 1 gives for each mass the mass loss rate at two epochs: at 88 Myrs (referred as models 1) and at 1095 Myrs for $1.6 M_{\odot}, 1032 \mathrm{Myrs}$ for $1.8 M_{\odot}$ and $824 \mathrm{Myrs}$ for $2.0 M_{\odot}$ (referred as models 5).

Figure 5 shows the helium profiles inside our models. As expected helium accumulates below the surface of the star. The helium peak lies in the vicinity of the first ionization region of helium and the overabundance increases with time. Helium is generally supported in the region of helium second ionization.
Table 1. Mass loss rates for models with $\Gamma=10^{-11} \mathrm{~g} \mathrm{~cm}^{-2} \mathrm{~s}^{-1}$ and $D_{\text {turb }}=2 \times 10^{5} \mathrm{~cm}^{2} \mathrm{~s}^{-1}$ (referred as S1) and $\Gamma=3 \times 10^{-11} \mathrm{~g} \mathrm{~cm}^{-2} \mathrm{~s}^{-1}$ and $D_{\text {turb }}=10^{6} \mathrm{~cm}^{2} \mathrm{~s}^{-1}$ (referred as S2). For the three masses, model 1 is 88 Myrs. Model 5 is $1095 \mathrm{Myrs}$ for $1.6 M_{\odot}, 1032$ Myrs for $1.8 M_{\odot}$ and 824 Myrs for $2.0 M_{\odot}$.

\begin{tabular}{c|cc|cc}
\hline \hline & \multicolumn{2}{|c|}{$\dot{\mathrm{M}}\left(\right.$ in $\left.10^{-14} M_{\odot} \mathrm{yr}^{-1}\right)$} & \multicolumn{2}{c}{$\dot{\mathrm{M}}\left(\right.$ in $\left.10^{-14} M_{\odot} \mathrm{yr}^{-1}\right)$} \\
\hline & \multicolumn{2}{|c|}{$\mathrm{S} 1$} & \multicolumn{2}{c}{$\mathrm{S} 2$} \\
Mass & model 1 & model 5 & model 1 & model 5 \\
\hline $1.6 M_{\odot}$ & 2.115 & 3.639 & 6.345 & 10.917 \\
$1.8 M_{\odot}$ & 2.334 & 5.943 & 7.002 & 17.829 \\
$2.0 M_{\odot}$ & 2.615 & 7.851 & 7.845 & 23.553 \\
\hline
\end{tabular}

On the contrary it rapidly diffuses out of the hydrogen ionization region which becomes hydrogen rich.

Figures 6 and 7 illustrate the impact of the wind flux and the turbulent diffusion coefficient on the helium accumulation. Figure 6 displays helium profiles for two $1.6 M_{\odot}$ models with the same turbulent mixing but different values of the windinduced helium flux and Fig. 7 shows helium profiles for two $1.8 M_{\odot}$ models with the same values of $\Gamma$ but different $D_{\text {turb }}$ values. The large helium values, close to one, obtained for the $1.8 M_{\odot}$ models with $D_{\text {turb }}=10^{5} \mathrm{~cm}^{2} \mathrm{~s}^{-1}$ are unrealistic and the atom test approximation is a very crude approximation in this context but it seems sufficient to illustrate the following results. The efficiency of the helium accumulation depends on both the wind strength and on the turbulent mixing efficiency; $\Gamma$ also acts on the position of the peak: it moves towards the surface with increasing $\Gamma$ values.

\subsubsection{Magnetic field and convection}

Our computations assumed that the magnetic field suppresses envelope convection. In this section we check how reasonable this assumption is for the models used. Despite the difficulty in determining the angular extent of the suppression of convection (cf. discussion in Sect. 2.1) it is interesting to check whether that assumption is reasonable at least in the region where such suppression is most likely to occur - the polar region, where the magnetic lines are vertical. For that we can compare the force exerted by buoyancy and the magnetic restoring force (see BCDGV). For the magnetic field to inhibit convection, one expects:

$$
\frac{\left|B^{2}\right| k^{2}}{\mu \rho} \geq-N^{2}
$$

where $\mu$ is the magnetic permeability, $\rho$ the density, $B$ the strength of the magnetic field, $k$ the characteristic wavenumber of the magnetic line distortion and $N$ the Brunt-Väissälä frequency:

$$
N^{2}=\frac{g}{H_{\mathrm{p}}}\left(\nabla_{\mathrm{ad}}-\nabla+\nabla_{\mu}\right)
$$

where:

$$
\nabla_{\mathrm{ad}}=\left(\frac{\partial \ln T}{\partial \ln P}\right)_{\mathrm{ad}} \quad \nabla=\left(\frac{\partial \ln T}{\partial \ln P}\right) \quad \nabla_{\mu}=\left(\frac{\partial \ln \mu}{\partial \ln P}\right) .
$$



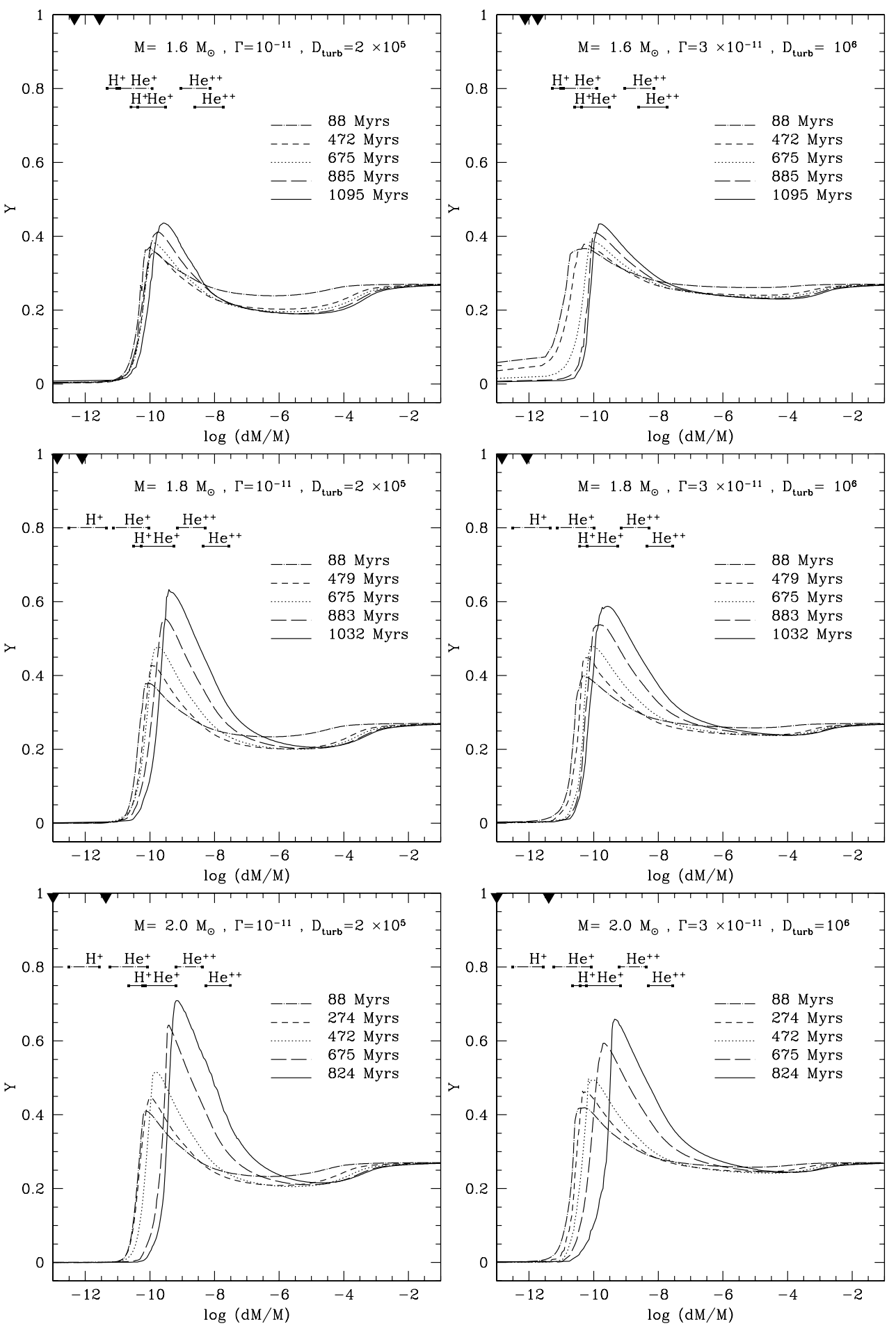

Fig. 5. Polar models including He diffusion and stellar wind: helium profiles at different evolutionary stages. The hydrogen and helium ionization regions are represented on each plot with the same convention as in Fig. 4. The solid triangles on the upper scale represent the depth where the optical depth is equal to 1 for respectively the youngest and the oldest model.

Generally, where the magnetic field is vertical the wavenumber $k$ may be approximated by the inverse of the pressure scale height $\left(k \sim 1 / H_{\mathrm{p}}\right)$.

To make easier the comparison between our results and those of Cunha (2002), we choose to compare $\left(\left|B^{2}\right| k^{2} c^{2}\right) /\left(\mu \rho \gamma g^{2}\right)$ and $\left(-N^{2} c^{2}\right) /\left(\gamma g^{2}\right)$ instead of $\left|B^{2}\right| k^{2} /(\mu \rho)$ and $\left(-N^{2}\right)$. Figure 8 (a and $\mathrm{b}$ ) shows these comparisons at three different ages for 1.6, 1.8 and $2.0 M_{\odot}$ models respectively without and with wind $\left(\Gamma=10^{-11} \mathrm{~g} \mathrm{~cm}^{-2} \mathrm{~s}^{-1}\right)$. The solid lines represent the buoyancy term and the dashed and dotted lines the magnetic term for two values of $B(1000 \mathrm{G}$ and $2500 \mathrm{G})$ as a function of the outer mass fraction.

We observe different shapes of the Brunt-Väissälä frequency profiles for models with and without wind (for 

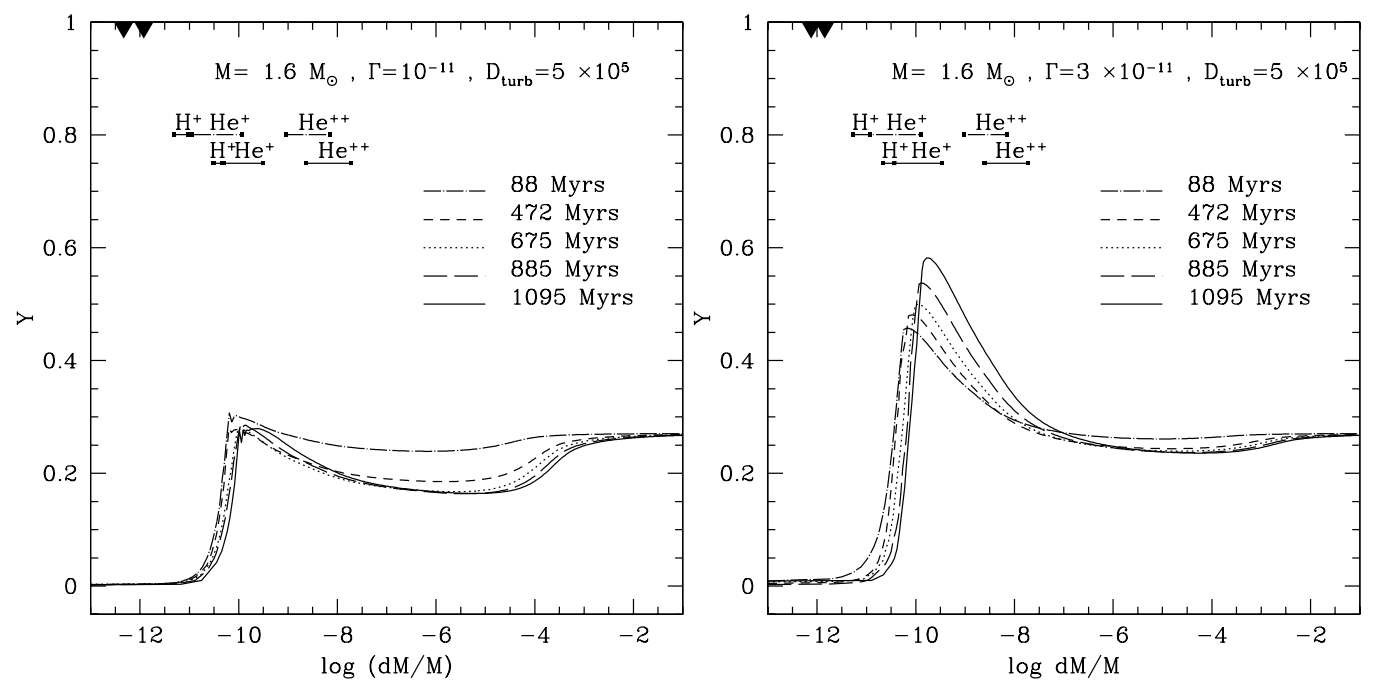

Fig. 6. Helium profiles in two $1.6 M_{\odot}$ polar models with the same value of the turbulent diffusion coefficient but different strength of the wind-induced helium flux. The solid triangles on the upper scale represent the depth where the optical depth is equal to 1 respectively for the youngest (on the left) and the oldest (on the right) models.
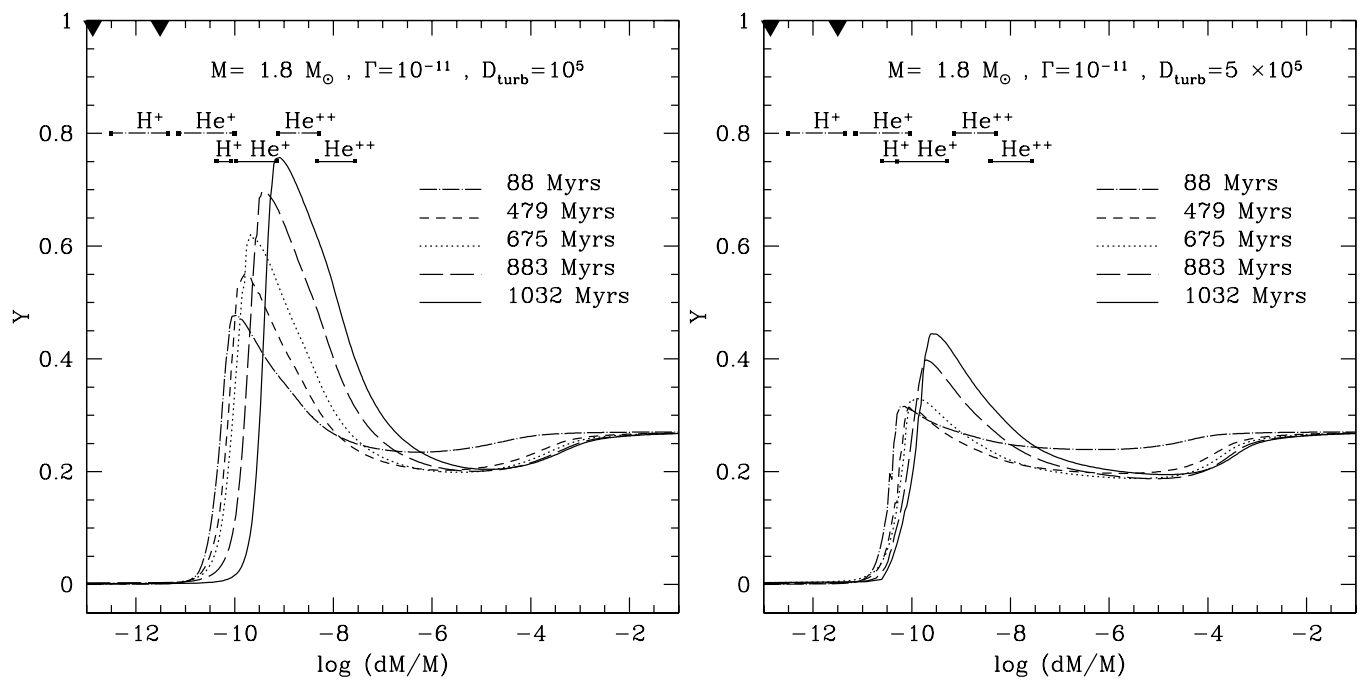

Fig. 7. Helium profiles in two $1.8 M_{\odot}$ polar models with the same value of the wind-induced helium flux but different value of the turbulent diffusion coefficient. The solid triangles on the upper scale represent the depth where the optical depth is equal to 1 respectively for the youngest (on the left) and the oldest (on the right) models. The large helium values close to one obtained for $D_{\text {turb }}=10^{5} \mathrm{~cm}^{2} \mathrm{~s}^{-1}$ is unrealistic but this comparison is done just to test the diffusion coefficient effects on the He accumulation.

$\log (\mathrm{d} M / M)$ between -9.5 and -10.5$)$. The negative peak observed in the profiles of $\left(-N^{2}\right)$ for models including wind results from to the strong helium gradients in the regions of helium accumulation.

Figures $8 \mathrm{a}$ and $\mathrm{b}$ show that for a given mass and a given magnetic field the magnetic term remains nearly constant with time. On the other hand, for a given mass, the relative importance of buoyancy increases during the stellar evolution which means that the magnetic field needed to suppress the surface convective region of a model increases with time. Figures 8a and $b$ also show that the buoyancy is more efficient in low mass models than in more massive models.

Quantitatively, we show that a $2500 \mathrm{G}$ vertical magnetic field is likely to suppress the surface convective regions of models with masses between 1.6 and $2.0 M_{\odot}$ during all their main sequence phase. A $1000 \mathrm{G}$ vertical magnetic field may suppress the surface convective zone only partially in a $1.6 M_{\odot}$ during its whole main-sequence phase. For models with masses greater than $1.8 M_{\odot}$, such a field is likely to suppress convection during the early stages of the main-sequence but might become unable to do it after a couple of hundred megayears.

Our conclusions are then the same as Cunha (2002): the convection seems to be easier to suppress in more massive stars than in low mass stars and it seems also easier to suppress in young stars than in evolved stars. From observational results, the magnitude of Ap stars magnetic field ranges typically from a few hundred to a few thousand Gauss. As a consequence some of these stars may have magnetic fields that are too small to suppress totally their polar surface convective regions during all their evolution. 

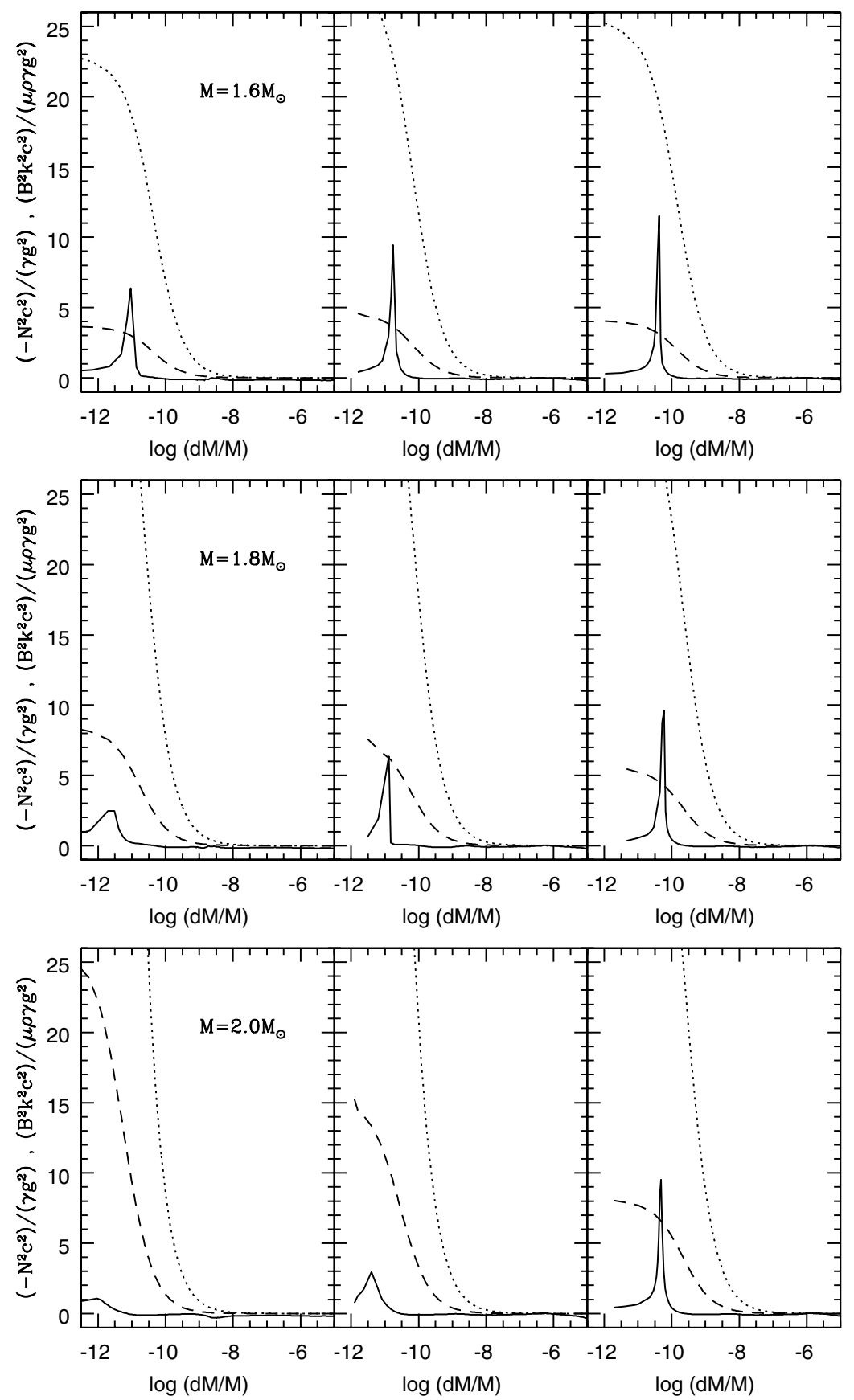

Fig. 8. a) Comparisons between the buoyancy term $\left(\left(-N^{2} c^{2}\right) /\left(\gamma g^{2}\right)\right.$, solid line $)$ and the magnetic field term $\left(\left(\left|B^{2}\right| k^{2} c^{2}\right) /\left(\mu \rho \gamma g^{2}\right)\right)$ for $B=1000 \mathrm{G}$ (dashed line) and $B=2500 \mathrm{G}$ (dotted line) in models including He settling but no wind. These comparisons are given as a function of the outer mass fraction at three different ages (upper panel (1.6 $M_{\odot}$ ): 0.05 Myrs, 675 Myrs, 1095 Myrs, middle panel (1.8 $\left.M_{\odot}\right)$ : 0.05 Myrs, 675 Myrs, 1047 Myrs, lower panel $\left(2.0 M_{\odot}\right)$ : 0.05 Myrs, 472 Myrs, 824 Myrs).

\subsection{Equatorial models}

We present now the results for equatorial models. Mass loss as well as convection are supposed to be suppressed by the magnetic field. Element segregation occurs but is reduced by the horizontal magnetic field as described in Sects. 2.2 and 3.4. We introduce in the computations a $2000 \mathrm{G}$ magnetic field. As element segregation may compete with turbulent mixing (if the magnetic field is not able to suppress it) we treat two cases: equatorial models are computed assuming no turbulent motions near the equator and equatorial models are computed including turbulent mixing.

\subsubsection{Models without turbulence}

The helium profiles of equatorial models without turbulence are nearly identical to the ones of polar models without wind (cf. Fig. 4). As expected $2000 \mathrm{G}$ magnetic field is actually unable to reduce significantly helium settling in the equatorial models. Similar models have been computed for stronger 

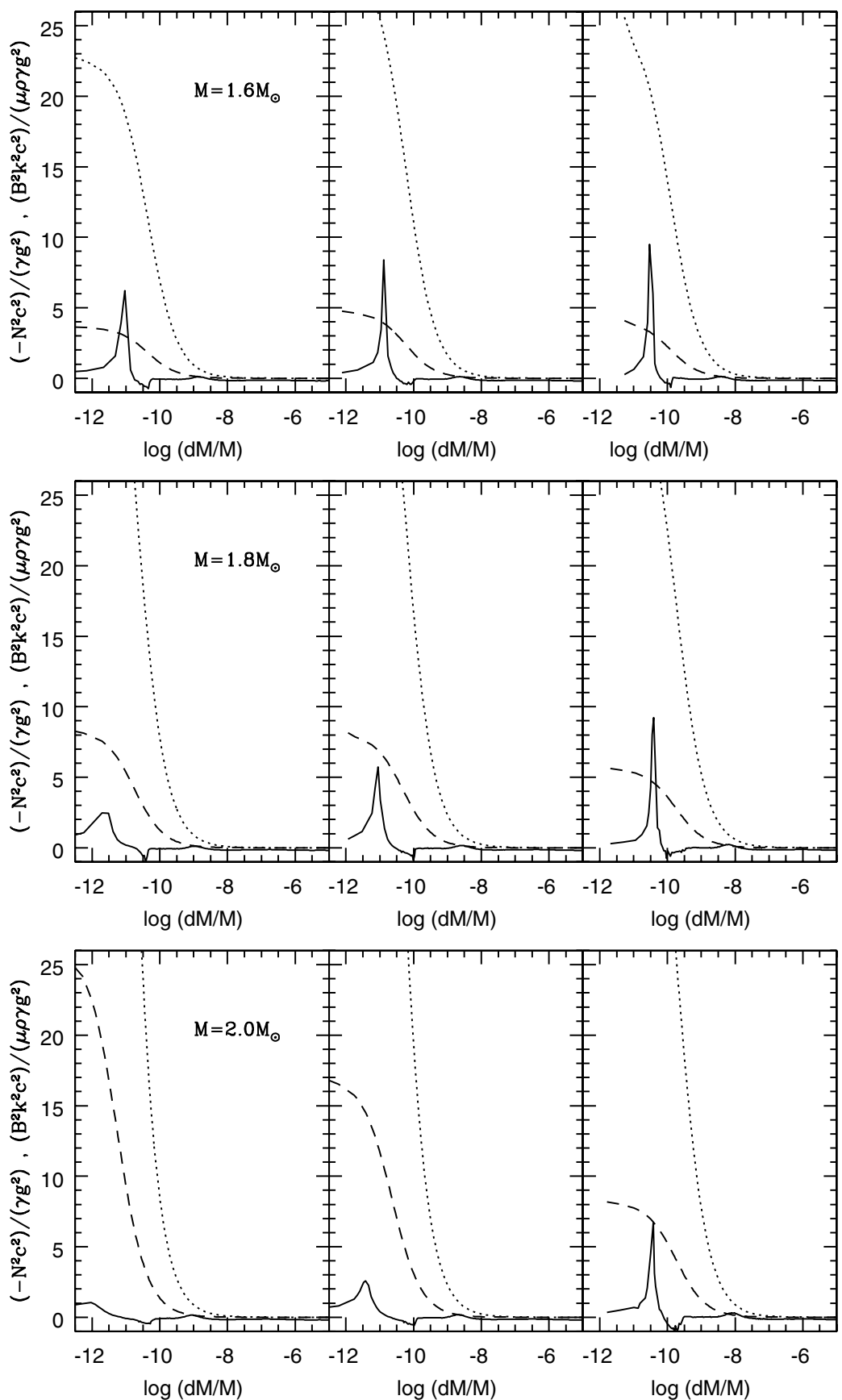

Fig. 8. b) Same figure as Fig. 8 a for models including He settling and stellar wind $\left(\Gamma=10^{-11} \mathrm{~g} \mathrm{~cm}^{-2} \mathrm{~s}^{-1}, D_{\text {turb }}=2 \times 10^{5} \mathrm{~cm}^{2} \mathrm{~s}^{-1}\right)$.

magnetic field: the He profiles are significantly modified only for magnetic field strength of the order of $10^{7}$ Gauss. According to the typical values of magnetic fields observed in roAp stars, we then expect helium settling to be hardly reduced in the equatorial regions.

\subsubsection{Models including turbulence}

For equatorial models with turbulence, the turbulent diffusion coefficient $\left(D_{\text {rot }}\right)$, which is a free parameter, is chosen large enough to inhibit helium settling, i.e. to prevent helium from falling down towards the central regions. Values as large as $10^{5}$ typically may allow the homogeneisation of the radiative interior. The chemical compositions of these equatorial models are then homogeneous.

\section{Discussion}

The chemical stratification, and, in particular, the abundance profiles of hydrogen and helium, is expected to play an important role in the excitation of the oscillations observed in roAp stars. The chemical profiles depend on a subtle interplay between several physical processes including helium settling, mass loss, turbulent mixing and the effect of the magnetic field on convection and microscopic diffusion. The purpose of this 
paper was to investigate on the effect produced by these physical processes on the helium stratification in models of roAp stars. We considered models with different degrees of mixing, as well as models with and without a wind. The models described in this paper will be used as input for pulsation models which will be discussed in a forthcoming paper (Cunha et al. 2005).

Because of their dipolar magnetic structure, the magnetic Ap stars are thought to have different structure at their magnetic poles (where the magnetic field is radially oriented) and equator (where the magnetic field is predominantly horizontal). Thus we modeled separately the polar and equatorial regions and we computed for each of them evolutionary models including different physics inputs taking into account the orientation of the magnetic field.

The polar models were computed assuming a radial magnetic field strong enough to suppress convective motions. Helium settling was introduced in the computations and we also studied the effects of a mass loss. Most of the turbulent motions were supposed to be suppressed by the magnetic field (as well as convective motions), only a mild mixing was allowed to take place with the occurrence of thermohaline instabilities.

In the absence of mass loss, both the helium and hydrogen ionization regions become rapidly helium-poor and hydrogenenriched because of element segregation. We expect this feature to increase the $\kappa$-mechanism of hydrogen (responsible for high frequency modes observed in roAp stars, Sect. 2) and stabilize the $\kappa$-mechanism of helium (responsible for low frequency modes observed in $\delta$-Scuti stars). As a consequence primarily high order modes, with frequencies typical of those observed in roAp stars, are expected to be excited in these models.

When a stellar wind competes with element segregation, the hydrogen ionization region becomes rapidly hydrogenenriched but an accumulation of helium takes place in the region where helium suffers its first ionization. We expect these models to show high order modes but also unstable low order modes excited in the helium ionization region, such as those observed in $\delta$-Scuti stars.

Equatorial models were computed taking into account the predominantly horizontal magnetic field. Given the difficulty in determining the extent to which convection is affected by a horizontal magnetic field, we assumed that convection was prevented at all latitudes. Helium settling was introduced in these models taking into account small corrections in the diffusion velocity due to the horizontal magnetic field. Following the theoretical and observational evidences for suppression of stellar wind at stellar magnetic equators, our equatorial models did not include stellar wind. We then studied two extreme cases: some models were computed assuming turbulent motions were suppressed and other models were computed assuming strong turbulent motions. Equatorial regions without turbulent motions are nearly identical to polar models without wind. We then expect the same kind of modes to be excited in these two kinds of models (i.e. primarily high order modes). Equatorial models including turbulent motions have homogeneous chemical composition. In such models we expect both high order and low order modes to be excited as predicted by BCDGV.
For a mode to be unstable in the composite model (taking into account the properties of both polar and equatorial regions), its growth rate must be positive in both polar and equatorial regions or the growth rate associated with the region where the mode is excited must overwhelm the damping rate associated with the region where the modes is stable. The stability of each model together with the stability of the composite models will be investigated in a forthcoming paper (Cunha et al. 2005).

Acknowledgements. S.T. was supported by grant POCTI/1999/ FIS/34549 approved by FCT and POCTI, with funds from the European Community programme FEDER and by ESA-PRODEX "CoRoT Preparation to exploitation I" through the grants C90135. S.V. acknowledges a grant from Institut universitaire de France. MC is supported by FCT-Portugal, through the grants BPD/8338/2002 and POCTI/CTE-AST/57610/2004.

\section{References}

Alexander, D. R., \& Ferguson, J. W. 1994, ApJ, 437, 879

Angulo C., Arnould M., \& Rayet M., (NACRE collaboration) 1999, Nucl. Phys. A, 656, 1,

http://pntpm.ulb.ac.be/Nacre/nacre.htm

Babel, J., \& Michaud, G. 1991, ApJ, 366, 560

Babel, J., \& Montmerle, G. 1997, A\&A, 323, 121

Balmforth, N. J., Cunha, M. S., Dolez, N., Gough, D. O., \& Vauclair, S. 2001, MNRAS, 323, 362

Brown D. N., Shore, S. N., \& Sonneborn, G. 1985, AJ, 90, 1354

Brown, A., Vealé, A., Judge, P., Bookbinder, J. A., \& Hubeny, I. 1990, ApJ, 361, 220

Cassinelli, J. P., \& MacGregor, K. B. 1986, in Physics of the Sun, ed. P. Sturrock, T. Holzer, D. Mihalas and R. Ulrich (Dordrecht: Reidel), 47

Cassinelli, J. P., \& Lamers, H. J. G. L. M. 1987, in Exploring the Universe with the IUE Satellite, ed. Y. Kondo (Dordrecht: Reidel), 13

Chapman, S., \& Cowling, T. G. 1970, The Mathematical Theory of Non uniform Gases (Cambridge: University Press), 3rd ed., 365

Charbonneau, P., \& MacGregor, K. B. 1995, ApJ, 454, 901

Charbonnel, C., Vauclair, S., \& Zahn, J.-P. 1992, A\&A, 255, 191

Cox, J. P. 1984, ApJ, 280, 220

Cunha, M. S. 2002, MNRAS, 333, 47

Cunha, M. S., Théado, S., \& Vauclair, S. 2005, in preparation

Cuntz, M. 1990, ApJ, 353, 255

Dolez, N., \& Gough, D. O. 1982, in Pulsations in Classical and Cataclysmic Variable Stars, ed. J. P. Cox, \& C. J. Hansen, 248

Dolez, N., Gough, D. O., \& Vauclair, S. 1988, in Helio and Asteroseimology, ed. J. Christensen-Dalsgaard, \& S. Frandsen, Proc. IAU Symp., 123, 291

dos Santos, L. C., Jatenco-Pereira, V., \& Opher, R. 1993, ApJ, 410, 732

Drake, S. A., Abbott, D. C., Bastian, T. S., et al. 1987, ApJ, 322, 902

Dupree, A. K., \& Reimers, D. 1987, in Exploring the Universe with the IUE Satellite, ed. Y. Kondo (Dordrecht: Reidel), 321

Elkin, V. G., Riley, J. D., Cunha, M. S., Kurtz, D. W., \& Mathys, G. 2005, MNRAS, accepted

Gelbmann M., Ryabchikova T., Weiss W. W., et al. 2000, A\&A, 356, 200

Girish, V., Seetha, S., Martinez, P., et al. 2001, A\&A, 380, 142

Gough, D. O., \& Tayler, R. J. 1966, MNRAS, 133, 85

Guzik, J. A., Kaye, A. B., Bradley, P. A., Cox, A. N., \& Neuforge, C. 2000, ApJ, 542, L57

Handler, G., \& Paunzen, E. 1999, A\&AS, 135, 57 
Holzer, T. E. 1987, in Circumstellar matter (Dordrecht: Reidel), Proc. IAU Symp., 122, 289

Holzer, T. E., \& MacGregor, K. B. 1985, in Mass loss from Red Giants, ed. M. Morris and B. Zuckerman (Dordrecht: Reidel), 229

Hubrig S., Kharchenko N., Mathys G., \& North P. 2000, A\&A, 355, 1031

Iglesias, C. A., \& Rogers, F. J. 1996, ApJ, 464, 943

Jatenco-Pereira, V., Opher, R., \& Yamamoto, L. C. 1994, ApJ, 432, 409

Kurtz, D. W. 1982, MNRAS, 200, 807

Kurtz, D. W., Martinez, P., \& Tripe, P. 1994, MNRAS, 271, 421

Landstreet, J. D. 1992, A\&ARv, 4, 35

Landstreet, J. D. 1998, A\&A, 338, 1041

Lanz, T., \& Catala, C. 1992, A\&A, 257, 663

Mathys, G., Hubrig, S., Landstreet, J. D., Lanz, T., \& Manfroid, J. 1997, A\&AS, 123, 353

Martinez, P., Kurtz, D. W., \& Kaufmann, G. 1991, MNRAS, 250, 666

Martinez, P., \& Kurtz, D. W. 1994, MNRAS, 271, 118

Michaud, G. 1986, in Hydrogen deficient stars and related objects, ed. K. Hunger et al. (Dordrecht: Reidel), 453

Michaud, G., Martel, A., \& Ratel, A. 1978, ApJ, 226, 483

Michaud, G., Martel, A., Montmerle, T., et al. 1979, ApJ, 234, 206

Michaud, G., Mégessier, C., \& Charland, Y. 1981, A\&A, 103, 244

Michaud, G., Tarasick, D., Charland, Y., \& Pelletier, C. 1983, ApJ, 269, 239

Montmerle, T., \& Michaud, G. 1976, ApJS, 31, 489

Moss, D. L., \& Tayler, R. J. 1969, MNRAS, 145, 217

North, P., Jaschek, C., Hauck, B., et al. 1997, Proceedings of the ESA Symposium 'Hipparcos - Venice '97', ESA SP-402, 239
Paquette, C., Pelletier, C., Fontaine, G., \& Michaud, G. 1986, ApJS, 61, 177

Richard, O., Vauclair, S., Charbonnel, C., \& Dziembowski, W. A. 1996, A\&A, 312, 1000

Richard, O., Théado, S., \& Vauclair, S. 2004, SoPh, 220, 243

Raybchikova, T. 1991, in Evolution of Stars: the Photospheric Abundance Connection, Proc. IAU Symp., 145, 149

Ryabchikova, T., Nesvacil, N., Weiss, W. W., et al. 2004, A\&A, 423, 705

Shibahashi, H. 1983, ApJ, 275, L5

Shore, S. N. 1987, AJ, 94, 73

Shore, S. N., Brown, D. N., \& Sonneborn, G. 1987, AJ, 94, 737

Shore, S. N., \& Brown, D. N. 1990, ApJ, 365, 665

Thomas, J. H., \& Weiss, N. O., ARAA, 42, 517

Underhill, A. B. 1983, ApJ, 268, L127

Vauclair, S. 1975, A\&A, 45, 233

Vauclair, S. 2004, ApJ, 605, 874

Vauclair, S., \& Dolez, N. 1990, in Progress of Seismology of the Sun and Stars, ed. Y. Osaki, H. Shibahashi, 399

Vauclair, G., Vauclair, S., \& Pamjatnikh, A. 1974, A\&A, 31, 63

Vauclair, S., Hardorp, J., \& Peterson, D. M. 1979, ApJ, 227, 526

Vauclair, S., Dolez, N., \& Gough, D. O. 1991, A\&A, 252, 618

Wade, G. A., Donati, J.-F., Landstreet, J. D., \& Shorlin, S. L. S. 2000, MNRAS, 313, 851

Willson, R. F., Lang, K. R., \& Foster, P. 1988, A\&A, 199, 25

Zahn, J.-P. 1993, in Astrophysical Fluid Dynamics, Les Houches Session LXVII, ed. J.-P. Zahn and J. Zinn-Justin 